\title{
Culture Collections and the Biotechnology Deal
}

\author{
Martin Sievers*, Gottfried Dasen, Tobias Wermelinger, Silvano Landert, and David Frasson
}

\begin{abstract}
Culture collections provide starting material for life science research, development and production. Especially in biotechnology, well characterised and pure microbial strains are essential for reproducible and safe bioprocesses. Culture collections also play a role as repositories of biological material for future applications and help to preserve biological diversity. In addition, they also maintain the know-how needed for more complex identification methods and help to develop new techniques. To enable culture collections to achieve higher quality standards, new certification guidelines for biological resource centres are currently being developed.
\end{abstract}

Keywords: Biological resource centres · CCOS · Culture Collection of Switzerland

\section{Introduction}

Microbial culture collections are an essential part of the infrastructure underpinning life sciences and biotechnology.

They collect and preserve cultivable microorganisms, replicable parts of these (e.g. genomes, plasmids, viruses, cDNAs), and also maintain databases containing molecular, physiological and structural information about these cultures. Good characterization of preserved microbial strains at genetic and proteomic level allows their use in biotechnological, agricultural, environmental and medical applications. By hosting these microorganisms, culture collections supply their users with verified and well documented starting material for their research. Biological resource centres of high quality that provide all legitimate users with access to industrially useful microorganisms will meet the future requirements of the life sciences and biotechnology (OECD: Biological Resource Centres-

${ }^{\star}$ Correspondence: Prof. Dr. M. Sievers Culture Collection of Switzerland c/o ZHAW

Einsiedlerstrasse 29

$\mathrm{CH}-8820$ Wädenswil

Tel. +41589345716

E-mail: martin.sievers@zhaw.ch
Underpinning the Future of Life Sciences and Biotechnology (http://www.oecd.org/ dataoecd/55/48/2487422.pdf).

Culture collections, and the associated biodiversity research, can point out the usefulness and importance of unique organisms and contribute to the preservation of biodiversity, preventing the loss of important genetic resources. In addition, collections are repositories of biological reference material, biological resources for the protection of intellectual property, and sources of public information.

"The rationale for establishing ex situ collections of microorganisms is based on the recognized role microorganisms play in the environment, not only as global players in the metabolism of nitrogen, phosphate, oxygen and carbon, but also as potential sources of problems for humans, animals and plants. Such organisms must be conserved and made readily available for research and utilisation in academia and industry" (Erko Stackebrandt, Biotech 2009, Isolation, Identification and Preservation of Microorganisms, ZHAW, CHWädenswil).

Deposition of a microorganism in a culture collection is free of charge and the procedure is shown in Fig. 1. Briefly, the depositor contacts a collection and is then invited to send his strain(s) to the collection. The strain is cultivated, submitted to stringent quality controls and preserved for instance by cryoconservation at $-196^{\circ} \mathrm{C}$ or lyophilisation. A sample is returned to the depositor for verification and after approval the strain is made available for sale. A deposit in at least two culture collections is required when a new species is described.

Biological resource centres are involved in a 'biotechnology deal' between their users and the providers of genetic resources. Benefits (monetary and/or non- monetary) are shared between users and providers of genetic resources based on mutually agreed terms, as defined in the Convention on Biological Diversity (CBD, http://www.cbd.int), ratified by most countries including Switzerland. Only the country providing genetic resources has access to its genetic resources and other countries or entities using these genetic resources have to share benefits arising from their use. In addition, access to and transfer of technologies that are relevant to the conservation and sustainable use of biodiversity shall be facilitated.

The Culture Collection of Switzerland (CCOS), located at the Zurich University of Applied Sciences in Wädenswil, was recently founded as a new repository of bacterial and yeast strains. The mission of the CCOS is to collect, administer and distribute well characterized bacteria and yeast strains with a Swiss background, and to act as a platform for know-how in the fields of identification, characterization and preservation of microorganisms. Special property strains and strains representing a broad biodiversity are collected, verified, catalogued and made available to a wider public. The Culture Collection of Switzerland promotes the efficient use of natural resources and makes microbial genetic resources more easily available for research and product development.

Identification of strains at CCOS is based on phenotypic features (protein profiles, fatty acid methyl ester profiles, biochemical properties) and sequencing of different genes such as ribosomal RNA, gyrase B and RNA polymerase subunit genes $r p o B$ or $r p o D$. The phylogenetic position of a bacterial isolate is verified by $16 \mathrm{~S}$ rDNA sequencing and alignment of sequences of closely related organisms. When differentiation of closely related species is not possible based on the 


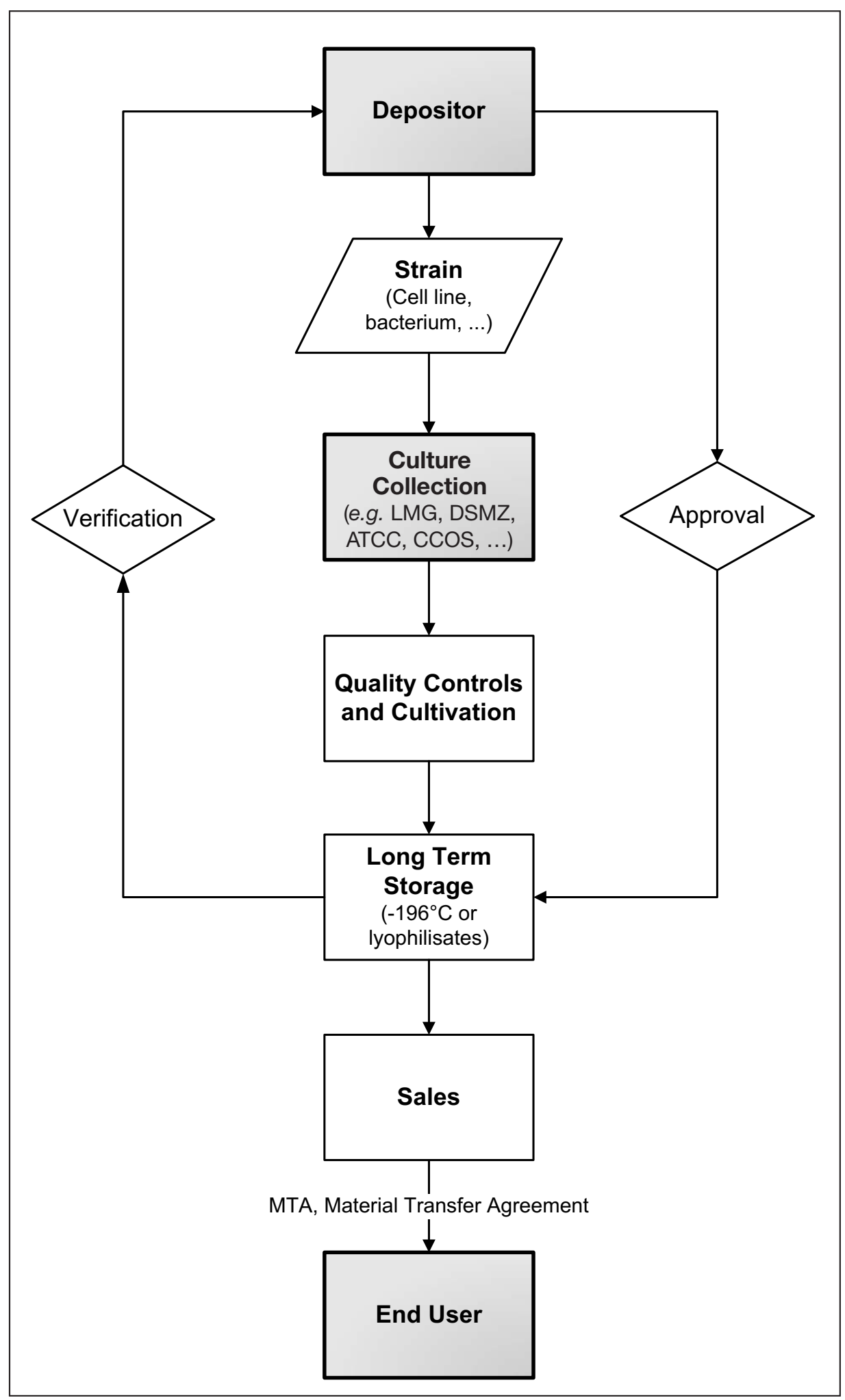

Fig. 1. Schematic representation of the deposition process of microorganisms in a public culture collection.

use of 16S rRNA sequencing, additional gene sequences such as gyrase B and RNA polymerase subunit genes $r p o B$ or $r p o D$ are used as molecular markers that provide better phylogenetic resolution. In over 100 Pseudomonas strains studied, the rpoD gene exhibited the highest number of polymorphic sites $(70.4 \%)$, followed by $g y r B$ $(51.8 \%)$ and $r p o B(44.4 \%))^{[1]}$ For species delineation, DNA-DNA hybridization to supplement DDH. ${ }^{[3,4]}$ For validation of such methods, strain collection representatives of the phylogenetic lineage of the new species described should be included in the study. ${ }^{[5]}$ Whole genome sequencing is the upcoming method for facilitation of taxonomic studies. Speed, resolution power, ease of use and accuracy, as well as sinking costs, recommend this method as the new gold standard for bacterial identification. The GEBA project (Genomic Encyclopedia of Bacteria and Archaea, http://www. jgi.doe.gov/programs/GEBA/index.html) aims to sequence at least the complete genome of the type strain of every bacterial species described so far. Genome sequencing is a likely candidate to replace DNADNA hybridization in the near future. ${ }^{[6]}$

Professional management of cultures is the basis for high quality research and development in biotechnology.

The CCOS would like to thank the FOEN, CTI in Berne, the Swiss Biotech Association, strain depositors, and the Swiss Industrial Biocatalysis Consortium for their help in building up the Culture Collection of Switzerland.

Received: September 6, 2010

[1] M. Mulet, J. Lalucat, E. García-Valdés, Environ Microbiol. 2010, 12, 1513.

[2] K. H. Schleifer, Syst. Appl. Microbiol. 2009, 32, 533.

[3] D. Gevers, P. Dawyndt, P. Vandamme, A Willems, M. Vancanneyt, J. Swings, P. De Vos, Phil. Trans. Roy. Soc. B 2006, 361, 1911.

[4] T. Adékambi, M. Drancourt, D. Raoult, Trends Microbiol. 2009, 17, 37.

[5] E. Stackebrandt, W. Frederiksen, G. M. Garrity, P. A. D. Grimont, P. Kämpfer, M. C. J. Maiden, X. Nesme, R. Rosselló-Mora, J. Swings, H. G. Trüper, L. Vauterin, A. C. Ward, W. B. Whitman, Int. J. System. Evol. Microbiol. 2002, 52, 1043.

[6] M. Richter, R. Rosselló-Móra, PNAS 2009, 106 , 19126.
(DDH) is currently the taxonomic standard and by definition, strains with DDH values of more than $70 \%$ under standardized conditions belong to the same species. Advantages and drawbacks of DDH are discussed by Schleifer. ${ }^{[2]}$ Investigators are encouraged to develop new methods such as multilocus sequence analysis (MLSA) and sequencing of the $r p o B$ gene encoding the $\beta$-subunit of the RNA polymerase 